\title{
La mirada imperial: Bingham y Machu Picchu
}

\author{
The Imperial Gaze: Bingham and Machu Picchu
}

Like a running blaze on a plain, like a flash of lightning in the clouds. We live in the flicker.

Joseph Conrad

JAVIER FLORES ESPINOZA

Pontificia Universidad Católica del Perú

javier.florese@pucp.pe

\section{RESUMEN}

En Perú, el descubrimiento de Machu Picchu ha resultado ser una manzana de la discordia desde que Hiram Bingham ascendiera la montaña y llegara por vez primera a la llacta incaica. La disputa librada por la Universidad de Yale y el gobierno peruano en torno a la posesión de los materiales arqueológicos que Bingham se llevó "temporalmente» del Perú, solo sirvió para agriar la discusión aún más. El centenario de su "descubrimiento" vio cómo las partes finalmente llegaron a un acuerdo, asi como la aparición de diversas publicaciones que o bien estudian estos materiales arqueológicos, o bien exploran su vida y obra. $Y$, sin embargo, a pesar de la firma del acuerdo, Bingham sigue siendo motivo de discusión en el Perú, el cual solo podrá resolver esta relación de amor-odio que mantiene con el explorador estadounidense una vez que acepte y asimile su pasado, pues lo que está en juego no es Bingham, sino la forma en que el Perú concibe y entiende su historia.

Palabras clave: Hiram Bingham, fotografí, Machu Picchu, memoria, turismo. 


\section{ABSTRACT}

In Peru, the discovery of Machu Picchu has proved to be a bone of contention ever since Hiram Bingham first climbed the mountain and reached the Inca settlement. The dispute between Yale University and the Peruvian government over the possession of the archaeological artefacts which Bingham "temporarily" removed from Peru only soured the discussion further. The centennial of Bingham's "discovery" saw both parties finally reach a settlement, as well as the publication of several books which either study these archaeological materials or explore Bingham's life and work. And yet Bingham still remains an issue in Peru, the agreement notwithstanding. This love-hate relationship with the American explorer will only be solved once Peru comes to terms with its past, for what is at stake is not so much Bingham, but how Peru conceives and understands its history.

Keywords: Hiram Bingham, Machu Picchu, Memory, Photography, Tourism.

$\mathrm{E}$ n los últimos años hemos visto cómo el mundo vive una explosión cada vez más incontenible del turismo. Sus efectos son visibles por doquier y hoy es ya usual encontrar a la población local quejándose de modo cada vez más ruidoso de estos visitantes, que cada vez resultan más y más indeseables. Los turistas, por su parte, tampoco dejan de quejarse de los lugares que visitan, como la Muralla China o la Torre Eiffel, por motivos muchas veces casi inverosímiles. Como era de esperar, Machu Picchu no podía escapar a esta tendencia. Tenemos así que se ha dicho que es un lugar «terrible» (i.e. pésimo, muy malo, desagradable) y de lo más decepcionante por, entre otras cosas, la falta de conectividad, el no poder usar Snapchat (lo que impide jactarse de él con las amistades en tiempo real) y por no ser otra cosa que un "tourist trap», esto es un lugar para turistas donde se les venden bienes y servicios con sobreprecio. ${ }^{1}$

1 Acerca de la población local harta del turismo, consúltese, por ejemplo, Will Coldwell, "Wish you weren't here: how the tourist boom—and selfies—are threatening Britain's beauty spots", The Guardian, 16.8.2018, https://www.theguardian.com/travel/2018/ aug/16/wish-you-werent-here-how-the-tourist-boom-and-selfies-are-threatening- 
Dejando de lado esta última crítica, llama la atención que los turistas se quejen de la falta de «modernidad» de Machu Picchu, pues una de las cosas que constituye su selling point es precisamente su condición de prístina ciudad perdida de la antigüedad.

Esta condición de «ciudad perdida» recientemente recobró actualidad con el centenario de su «descubrimiento» en 2011, con motivo del cual fueron apareciendo diversas publicaciones sobre el tema, las cuales fueron esencialmente de tres tipos: investigaciones arqueológicas del yacimiento, el catálogo de las piezas devueltas por la Universidad de Yale inclusive; ${ }^{2}$ estudios de la historia de Machu Picchu después de la caída de los incas, ${ }^{3}$ $y$, en menor medida, estudios que giran en torno a Hiram Bingham y su obra. Aquí me voy a ocupar de dos de estos últimos tipos de estudio.

El lugar natural donde iniciar este examen indudablemente es con Bingham mismo, cuyo libro Lost city of the Incas ha recibido una nueva y hermosa reedición en Inglaterra, precedida esta vez por una introducción a cargo del explorador Hugh Thomson, quien también recorrió la zona de Vilcabamba. ${ }^{4}$ El relato de Bingham sigue siendo cautivante, pero incluso con una lectura superficial resulta evidente, como señala Thomson en su introducción, que se trata de una mirada retrospectiva escrita cuarenta años después de que llegara por primera vez al asentamiento incaico, en lo que a la postre sería lo único que hizo en su vida que le permitiría alcanzar una fama duradera. Sucede que la carrera de Bingham como académico no fue precisamente brillante y su carrera política llegó a su fin rápidamente con un voto de censura en el Senado estadounidense. Tal vez en otro caso se le habría olvidado. Pero Bingham sin duda fue

britains-beauty-spots (consultado por última vez 8.11.2018). Sobre los turistas quejándose de los «tourists traps» véase Claudia Cuskelly, "No snapchat, too ugly \& boring: Tourists' bizarre complaints about world famous landmarks», 27.9.2016, https:// www.express.co.uk/travel/articles/714825/tourists-bizarre-complaints-world-famouslandmarks-tripadvisor-big-ben-machu-picchu (consultado por última vez 8.11.2018), que es de donde provienen las citas.

2 Por ejemplo, Burger y Salazar, 2008; Embajada de los Estados Unidos de América 2011 (el catálogo de las piezas devueltas por Yale); o Verano 2003, entre otros.

3 Mould de Pease 2003; Vilela y de la Puente 2011.

4 Thomson 2001. 
un gigante (literalmente: medía $1.95 \mathrm{~m}$ ) carismático y seductor, a cuyo encanto pocos escapaban. Fue precisamente gracias a esto que logró hacer fortuna casándose con Alfreda Mitchell, la heredera de la fortuna de la joyería Tiffany. Y solo así se explica que Luis E. Valcárcel, al enterarse del fallecimiento del explorador con quien antes había chocado, dijera simplemente que "Fue un gran hombre». ${ }^{5}$ Thomson evidentemente también siente gran admiración por Bingham, y lo mismo sucede con Christopher Heaney, quien ha dedicado un volumen íntegro a biografiar al joven explorador. ${ }^{6}$

Cradle of Gold. The Story of Hiram Bingham, a Real-Life Indiana Jones, and the Search for Machu Picchu, de Heaney, es una biografía muy ágil que apareció originalmente en inglés en 2010, y en español en 2012 bajo el título algo incomprensible de Las tumbas de Machu Picchu. El lector haría bien en advertir que, si bien se trata esencialmente del mismo libro, hay diferencias entre ambas ediciones. Heaney señala en su prólogo que su libro une tres hilos argumentales, uno que explica la lucha de los españoles contra los Incas de Vilcabamba, otro que examina cómo eran las exploraciones al comenzar el siglo XX y las que Bingham lideró, y un tercer hilo que examina qué significa hoy en día lo que «descubrió». Podríamos resumir esto preguntándonos con Heaney: «¿Quién puede poseer e interpretar el pasado indígena?». Si bien ambas ediciones hacen esto, la original en inglés es mucho más personal e incluye un capítulo —el último - suprimido en la edición en español, en el cual el autor se insertó a sí mismo en su propia narrativa y homenajeó a un difunto amigo. ${ }^{7}$ A cambio del capítulo suprimido, la edición peruana agrega otro nuevo con el cual Heaney actualiza su libro y lo extiende para incluir

5 Heaney 2010: 214.

6 El grueso del libro se ocupa de la vida de Bingham desde su infancia hasta sus años como explorador. Es poco lo que dice de él después de la Primera Guerra Mundial y, en general, lo que sí se expone está en función al Perú.

7 Heaney 2010: XIII. Usé la primera edición en inglés (2010) y luego hice un cotejo no exhaustivo con la edición en español. La cita proviene de la primera edición en inglés y la traducción es mía, lo que explica la diferencia con respecto a la edición en español, donde el traductor prefirió colocar "contar» mientras que yo mantuve el original «interpret». 
la resolución del diferendo entre el Perú y la Universidad de Yale en torno a la devolución de las piezas de Machu Picchu.

La imagen de Bingham que emerge del estudio de Heaney no es muy agradable que digamos, incluso si nos concentramos únicamente en su vida hasta el ingreso de los Estados Unidos a la Primera Guerra Mundial. Lo que parece haber primado en él desde muy joven fue el deseo de destacar y de ascender a como diera lugar, así como una gran ansia de vivir aventuras, lo que se manifestó tempranamente en un intento de fuga a los doce ańos. ${ }^{8}$ No parece, en cambio, haber estado muy interesado en los otros, ni haber sido muy empático. ${ }^{9}$ De hecho, Bingham llegó a ser tan egoísta e inescrupuloso que no solo buscó siempre acaparar todo el crédito para sí mismo —algo que el joven historiador Philip Ainsworth Means sufriría en carne propia—,${ }^{10}$ sino que además fue acusado públicamente de plagio una vez, en tanto que otro caso comprobado en su tesis de maestría pasó inadvertido. ${ }^{11} \mathrm{Su}$ arrogancia era tan grande que incluso emprendió expediciones arqueológicas sin realmente tener más que un conocimiento mínimo de arqueología, ${ }^{12}$ lo que explica el que se haya usado al fuego como método de limpieza de Machu Picchu, y que los trabajos de búsqueda se llevaran a cabo sin mayor supervisión, lo que permitió que sus «ayudantes» nativos incluso quemaran los envoltorios de algunas momias para espantar a los murciélagos de la cueva en donde se encontraban. ${ }^{13}$ Pero tal vez el error más grande de todos lo cometió, no en Machu Picchu ni con los restos arqueológicos, sino en la quebrada de Ayahuacco, en las afueras del Cuzco, donde su expedición descubrió huesos aparentemente humanos junto a un tosco muro de piedra. A Bingham le pareció que tenían unos dos mil años, pero Isaiah Bowman, el geógrafo de la expedición, sostuvo primero que su contexto

8 Heaney 2010: 14.

9 Ib.: 23 .

${ }^{10}$ Ib.: 206.

${ }^{11}$ Ib.: 22.

${ }^{12}$ En esto se encuentra en buena compañía, pues lo mismo hizo John V. Murra en Huánuco Pampa, con resultados cuestionables, por decir lo menos. Al respecto, consúltese Barnes 2013.

${ }^{13}$ Heaney 2010: 184. 
indicaba unos 30,000 años de antigüedad, y luego que en realidad eran 10,000 años más antiguos. Al final resultó que ni siquiera eran huesos humanos, sino de un bovino. ${ }^{14}$

Llama por ello poderosamente la atención que la posteridad haya tratado tan generosamente a un intelectual de carrera académica tan mediocre como Bingham. No son muchos los descubridores de «ruinas» cuyos nombres forman parte de la cultura popular, y son mucho menos los que han servido de inspiración para un personaje tan célebre como Indiana Jones. ${ }^{15}$ De hecho, es muy probable que Heinrich Schliemann sea el único descubridor de un yacimiento arqueológico más famoso que Bingham. Parte de la respuesta a este enigma sin duda yace en el momento en que su «descubrimiento» tuvo lugar, pues se dio cuando Occidente buscaba «descubrir» y «llegar primero» a los más remotos rincones del mundo; igualmente importantes fueron la habilidad de Bingham para construir redes de apoyo y el papel que la National Geographic Society y su revista tuvieron en la difusión de este hallazgo y en el financiamiento de las expediciones de 1912 y 1915. El libro de Amy Cox Hall, Framing a Lost City. Science, Photography, and the Making of Machu Picchu, ${ }^{16}$ ayuda a responder esta interrogante.

La exposición de Hall comprende seis capítulos además de la introducción. En primer lugar examina cómo a través de la escritura de cartas, Bingham fue construyendo una red de apoyo y respaldo que «le permitió ver». ${ }^{17}$ A continuación examina el coleccionismo de la expedición, al que denomina la "visión del huaquero», para pasar luego a la parte más sustancial del texto, donde examina la materialización de las figuraciones científicas iniciales como hechos representacionales que se movían, explorando sucesivamente las fotografías que popularizaron a Machu Picchu y lo convirtieron en un descubrimiento (cap. 3), la forma

\footnotetext{
${ }^{14}$ Heaney 2010: 80-81; Hall 2017: 199.

${ }^{15}$ Heaney (2010: XI-XII) toca este punto en la edición en inglés, pero en la edición en espańol se eliminó la referencia a Indiana Jones no solo en el título, sino también en el texto.

${ }^{16}$ Hall 2017.

${ }^{17}$ Ib.: 20.
} 
en que estas fueron recibidas tanto en los EE.UU. como en el Perú (cap. 4), y los estudios de antropología física realizados por la expedición de Yale en fotografías que casi no circularon porque su temática - el degenerado indio actual — "no celebraba[ba] el glorioso pasado inca» (cap. 5). ${ }^{18}$ El libro se cierra, una vez más, con una revisión del conflicto entre el Perú y la Universidad de Yale.

Según Hall, para realizar su descubrimiento, Bingham efectuó un doble movimiento que involucró la construcción de una red epistolar de sustento y el recurso a la ciencia. La primera, una red construida a partir de la escritura de cartas, le permitió construir una red de respaldo con la cual recabar fondos para buscar ruinas incaicas, ascender al Coropuna y efectuar levantamientos topográficos. Esto aseguró el financiamiento y equipamiento de la expedición, pero además le proporcionó información (recibida de Clements R. Markham y Julio C. Tello, por ejemplo) y una legitimación científica que le empoderó y le permitió ver a Machu Picchu como algo "perdido». Las técnicas y tecnologías empleadas a su vez reforzaron la naturaleza científica de la expedición, lo que permitió que su coleccionismo se diferenciara del de los huaqueros (a pesar de que eran muy parecidos) y que el saqueo de tumbas se convirtiera en recolección científica. ${ }^{19}$ Pero sobre todo «ayudaron a mitologizar Machu Picchu [convirtiéndolo] en una ciudad perdida... [y a] materializarlo... tanto como un vestigio de la raza inca y como un descubrimiento científico». ${ }^{20}$

La cámara fotográfica fue la herramienta científica más importante para la expedición — de hecho, «El descubrimiento era las fotografías»—, ${ }^{21}$ no solo porque esta era un instrumento de verificación que proporcionaba evidencias cruciales con que legitimar la antropología, o porque fuese un testimonio/testigo que pretendía producir una realidad objetiva, sino además porque permitió que la visión de Bingham se materializara y circulara. Mas como posteriormente admitiría, él no fue realmente consciente de lo que había logrado sino hasta que vio sus fotos publicadas

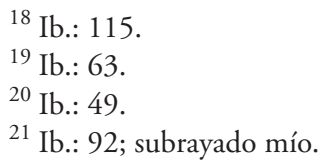


en National Geographic. ¿Cuál era esta visión y cuál su logro? Las fotos de Bingham constituían una narrativa de viaje y un acto de ver que enfatizaba magníficamente lo remoto y perdido, plasmando al mismo tiempo una «estética del descubrimiento que hizo que Machu Picchu fuera algo seguro y consumible». ${ }^{22}$ Pero esta estética y su acto de ver al mismo tiempo "requería de cierta ceguera»: los habitantes actuales de Machu Picchu eran prescindibles porque no eran sino los restos degradados de un pasado glorioso ${ }^{23}$ y por lo mismo, en general, se hicieron invisibles. Así, las fotos incluidas en el célebre artículo «In the Wonderland of Peru» (1913) mostraban una ciudad perdida descubierta por la ciencia que parecía levantarse en medio de un entorno despoblado, con lo cual el paisaje era «conquistado y poseído para Yale, la ciencia y National Geographic», ${ }^{24}$ y sobre todo para Bingham, quien finalmente logró alcanzar la aventura y la gloria que tanto había buscado desde niño.

Sin embargo, todo esto nos permite entrever por qué Bingham ganó fama y reconocimiento en ese entonces, no así porqué esta aún perdura. Algunas pistas tenemos. Según Hall, la fotografía permite estabilizar los hechos al oscurecer instantáneamente la construcción cultural y producir una realidad objetiva. ${ }^{25}$ Pero cuando estas comienzan a difundirse, su significado y contenido informativo se desplazan tan pronto quedan insertas dentro de diversos marcos narrativos, ${ }^{26}$ lo que provoca su inestabilidad. Fue así como dichas fotos fueron adquiriendo un nuevo significado una vez que comenzaron a circular en el Perú, el de la fuente o esencia de la nación peruana. Su publicación parcial en la revista Ilustración Peruana en 1912, o las fotografías que José Gabriel Cosio incluyera en el artículo que publicó ese mismo año, mostraron esta transformación al plasmar «una comunidad histórica imaginada con Machu Picchu al centro» gracias a «la circulación y traducción» de sus

${ }^{22}$ Ib.: 101.

${ }^{23}$ Ib.: 134 .

${ }^{24}$ Ib.: 111 .

${ }^{25}$ Ib.: 72 .

${ }^{26}$ Ib.: 85 . 
fotografías. ${ }^{27}$ A pesar de los años transcurridos desde entonces, Machu Picchu todavía conserva dicha posición central en la narrativa peruana. ${ }^{28}$ De ahí la profunda relación de amor-odio que el Perú mantiene con Bingham, la cual refleja las contradicciones y grietas geológicas de la sociedad peruana. La pugna por la devolución de las piezas extraídas de Machu Picchu avivó esto, y podría pensarse que la fijación peruana en Bingham amainará ahora que han sido devueltas, pero lo más probable es que ella perdure mientras los peruanos no confrontemos $-\mathrm{y}$ derrotemos- a nuestros demonios interiores.

\section{BIBLIOGRAFÍA}

Barnes, Monica. 2013. «John Victor Murra, arqueólogo accidental: de Cerro Narrío a Huánuco Pampa». En Henry Tantaleán y César Astuhuamán (eds.). Historia de la arqueología en el Perú del siglo XX. Lima: Instituto Francés de Estudios Andinos, Institute of Andean Research, 551-574.

Bingham, Hiram. 1913. «In the Wonderland of Peru». National Geographic, vol. XXIV, no. 4, abril, 387-573.

. 1977. Machu Picchu: la ciudad perdida de los incas. Santiago de ChileMadrid: Zig-Zag, Rodas. 2002. Lost City of the Incas. Londres: Weidenfeld \& Nicolson.

Burger, Richard L., y Lucy C. Salazar (eds.). 2008. Machu Picchu: Unveiling the Mystery of the Incas. New Haven: Yale University Press.

Embajada de los Estados Unidos de América. 2011. Machu Picchu. Catálogo de la colección. Machu Picchu. Collection Catalogue. Lima: Embajada de los Estados Unidos de América.

Hall, Amy Cox. 2017. Framing a Lost City. Science, Photography, and the Making of Machu Picchu. Austin: University of Texas Press.

Heaney, Christopher. 2010. Cradle of Gold. The Story of Hiram Bingham, a Real-Life Indiana Jones, and the Search for Machu Picchu. Nueva York: Palgrave Macmillan. . 2012. Las tumbas de Machu Picchu. La historia de Hiram Bingham y la buisqueda de las últimas ciudades de los incas. Lima: Pontificia Universidad Católica del Perú.

${ }^{27}$ Véase Hall 2017: 105-110 (la cita en la p. 110). Las referencias bibliográficas de Ilustración Peruana y de Cosio aparecen en las pp. 250 y 245, respectivamente.

${ }^{28}$ Hoy probablemente solo el Señor de Sipán ocupa un lugar comparable al de Machu Picchu en el imaginario peruano, pero se ve disminuido en comparación por la falta de un contexto igual de espectacular. 
Mould de Pease, Mariana. 2003. Machu Picchu y el código de ética de la Sociedad de Arqueología Americana: una invitación al diálogo intercultural. Lima: Consejo Nacional de Ciencia y Tecnología, Pontificia Universidad Católica del Perú, Instituto Nacional de Cultura y Universidad Nacional de San Antonio Abad del Cusco.

Thomson, Hugh. 2001. The White Rock. An Exploration of the Inca Heartland. Londres: Weidenfeld \& Nicolson. . 2002. "Introduction». En Bingham, Hiram. 2002. Lost City of the Incas. Londres: Weidenfeld \& Nicolson, 1-18.

Verano, John W. 2003. «Human Skeletal Remains from Machu Picchu. A Reexamination of the Yale Peabody Museum's Collections». En Richard L. Burger y Lucy C. Salazar (eds.). The 1912 Yale Peruvian Scientific Collections from Machu Picchu. Human and Animal Remains. New Haven: Yale University Publications in Anthropology, no. 85, 65-117.

Vilela, Sergio, y José Carlos de la Puente. 2011. El último secreto de Machu Picchu. Quién es dueño de la ciudadela de los incas. Lima: Punto y Coma Editores.

Fecha de recepción: 6/VI/2019

Fecha de aceptación: 20/VIII/2019 